\title{
Las mujeres como protagonistas del fanfiction hispanohablante
}

\author{
Women as protagonists of Spanish-speaking fanfiction
}

\author{
CRISTINA DE LOS Ríos IZQUIERDo \\ Universidad Complutense de Madrid
}

Recibido: $18-2-2021$

Aceptado: 19-7-2021

doi: https://doi.org/10.20318/femeris.2021.6402

Resumen. Este artículo aborda la figura de la mujer hispanohablante como escritora de fanfiction (ficción escrita por aficionados de una serie de televisión, película, etc. y con sus personajes) en algunas comunidades de fans (fandoms). El gran número de escritoras mujeres en los fandoms de los medios de comunicación ha feminizado tanto la actividad de escribir fanfiction como las propias comunidades de fans. Por lo tanto, los objetivos concretos de esta investigación son: en primer lugar, describir a las escritoras hispanohablantes de fanfiction -¿quiénes son?, ¿dónde están?, ¿qué hacen?-; y, en segundo lugar, explicar la feminización del fanfiction y de los fandoms a través, principalmente, de las temáticas predominantes. La metodología utilizada es mixta, cualitativa y cuantitativa, con el fin de encontrar un procedimiento adecuado mediante la triangulación en la recolección de datos de distintas fuentes primarias obtenidas ex profeso para la investigación. Los datos cuantitativos se han obtenido a partir de un cuestionario de elaboración propia, realizado a fans de los medios de comunicación, y los cualitativos, a partir de entrevistas personales a personas que crean contenidos de distintos fandoms. Las conclusiones llevan a considerar el fanfiction como un lugar de reunión para minorías que normalmente no han encontrado representación en los medios de comunicación y que, gracias a este espacio, han creado sus propios canales para comunicarse y transmitir historias. De esta manera, han conseguido sentirse representadas a través de una temática centrada, principalmente, en las relaciones interpersonales.

Palabras clave: fanfiction; fandom; mujeres creadoras; fenómeno fan; ship; slash.

Abstract. This study addresses how Spanish-speaking women write fanfiction (fiction written by fans of, and featuring characters from, a particular TV series, film, etc.) in some communities of fans (fandoms). The high number of female writers in fandoms in the media have feminized both the activity of writing fanfiction and the communities of fans itself. The goals of this research are: first, to describe the Spanish-speaking female fanfiction writers. Who are they? Where are they? What do they do? And, secondly, to explain how the predominant topics are making fanfictions and fandoms more feminine. The methodology used is mixed, qualitative and quantitative, trying to find the proper process through the triangulation in the data collection of different primary sources obtained expressly for this research. The quantitative data originates from a survey done with media fans and conceived specifically for this study. The qualitative data comes from personal interviews conducted with content creators of different fandoms. The outcomes show that fanfiction is a place of gathering for minorities who have usually not found themselves represented in media. And that, thanks to 
fandoms and fanfictions, have created their own communication channels to broadcast stories. In this way, they have achieved feeling represented through a thematic focused, mainly, in interpersonal relationships.

Keywords: fanfiction; fandom; women creators; fan phenomenon; ship; slash.

\section{Introducción}

Los fanfictions, fanfics o fics son historias o relatos escritos por fans, normalmente, en el contexto de un fandom ${ }^{1}$ o fandoms específicos, teniendo como base un producto ya existente -una serie, una película, un libro, un personaje... o, incluso, personas reales-.

Los trabajos literarios derivados no son algo nuevo y forman parte de la vida literaria desde su nacimiento. Sin embargo, los antecedentes del fanfic se pueden encontrar de la mano de los primeros fans conocidos, los Sherlockians ${ }^{2}$ y los Janeites ${ }^{3}$, quienes empezaron a agruparse en comunidades en el siglo XIX, y los fans de la ciencia ficción, ya bien entrado el siglo XX. El escritor y amigo de Sir Arthur Conan Doyle, James M. Barrie, publicó entre 1891 y 1893 tres relatos basados en el personaje de Sherlock Holmes. En 1913 se publicó Old Friends and New Fancies, de Sybil G. Brinton, una novela que es una precuela de distintas obras de Jane Austen. Y entre los años 30 a 50 del siglo XX, en plena Edad de Oro de la Ciencia Ficción y con un gran desarrollo de las agrupaciones de fans, nacieron los fanzines ${ }^{4}$, creados por los propios fans. En ellos se publicaban principalmente relatos y dibujos u otro tipo de representaciones artísticas, basados en la ciencia ficción. Algunos de estos fanzines fueron Amazing Stories y Astounding Science Fiction, y entre esos fans escritores encontramos, entre otros, a Julio Verne, Edgar Allan Poe, H. G. Wells e Isaac Asimov.

Los fanzines fueron el principal hogar del fanfiction durante mucho tiempo, incluido el caso del fandom por excelencia, el de Star Trek (Star Trek: The Original Series, NBC, 1966-1969), donde seguramente nacieron estos relatos como hoy los conocemos. Spockanalia -el primer fanzine de Star Trek, considerado el primer fanzine moderno- nació en 1967 repleto de fanfiction, fanart ${ }^{5}$ y otros productos hechos por fans. Esos fans estuvieron altamente estereotipados y denigrados durante años; recibían el nombre de trekkies y eran considerados personas sin vida propia, con problemas de sobrepeso y obsesionadas con cada pequeño detalle de la serie (Jenkins, 2010: 21-24), tanto es así que, en muchos casos, intentaban ocultar o minimizar su identidad como fans (Kozinets, 2001: 24-26). Fiel reflejo de este estereotipo, y de que se ha mantenido con el paso de los años es, por ejemplo, el personaje de Jeff Albertson -el dependiente de la tienda de

\footnotetext{
${ }^{1}$ Conjunto de personas unidas por un gusto común, con un vocabulario propio, actividades propias, sin límites estrictos, pero sí con normas de conducta aceptadas y respetadas, que gira alrededor de una afición.

${ }^{2}$ Fans y admiradores de los productos culturales protagonizados por el personaje de Sherlock Holmes.

${ }^{3}$ Fans y admiradores de la obra y la figura de la escritora Jane Austen.

${ }^{4}$ Revistas de escasa tirada y distribución hechas con pocos medios.

${ }^{5}$ Cualquier representación artística hecha por un fan sobre un producto ya existente, los más típicos son pinturas o dibujos y también imágenes editadas.
} 
cómics, en inglés Comic Book Guy- de la serie Los Simpson (The Simpsons, 20th Century Fox Television, 1989-actualidad).

Resulta significativo que, aunque el fan cómico y el fan psicótico por lo general se representan como masculinos, a pesar de que suelen ser hombres con un género neutro, asexuales o impotentes, la fan erotizada es casi siempre femenina (...); el lado femenino del mundo de los fans se manifiesta en las imágenes de adolescentes gritando que intentan arrancarles la ropa a los miembros de The Beatles... (Jenkins, 2010: 28)

Sin embargo, y contraviniendo la representación popular, las mujeres fans no solo eran groupies $^{6}$. En su artículo Spock among the women, publicado en 1986 en el periódico The New York Times, la académica y novelista Camille Bacon-Smith ya comentaba que la de Star Trek era una comunidad predominantemente femenina: "For the past 10 years, at least, women have accounted for over 90 percent of the writing and graphic arts and for almost all the editing of the 'Star Trek' fan publications" (Bacon-Smith, 1986) ${ }^{7}$. De hecho, en su libro Enterprising Women: Television Fandom and the Creation of Popular Myth, la autora hace un estudio de la amplia comunidad de fans femeninas que escriben sobre Star Trek y otras series de televisión.

De la misma manera, el investigador del fenómeno fan Henry Jenkins, en su libro Piratas de Textos, publicado en 1992, escribía que la mayor parte de los fandoms de los medios de comunicación están compuestos "principalmente por mujeres, en su mayoría blancas y de clase media” (Jenkins, 2010: 12), al igual que eran mujeres la mayoría de los fans creadores, entre los que se encontraban las escritoras de fanfiction.

Este tema también es tratado en profundidad, desde diversas pero cercanas perspectivas por otras académicas y autoras como Patricia Frazer Cordero, Sara Gwenllian Jones, Diana L. Veith y Joanna Russ, siendo esta última quien escribió en 1985 su ensayo Pornography By Women For Women, With Love, en el cual hacía un primer e importante análisis feminista sobre el $\operatorname{slash}^{8}$ sentando las bases del debate posterior (Francis y Piepmeier, 2011).

Por lo tanto, desde los años 70 las mujeres fans -y, sobre todo, las escritoras- se han ido convirtiendo en las cabezas visibles de un buen número de comunidades de fans. No solo en el mundo anglosajón, sino también en el hispanohablante. En todo el mundo, hay escritoras de fanfic ampliamente reconocidas y admiradas dentro de sus respectivas comunidades de fans e, incluso, la popularidad de algunas de ellas ha traspasado el propio fandom. Sin embargo, aunque la idea de ser fan cada vez está más asimilada y reconocida dentro de la sociedad actual, todavía hay muchos estereotipos que superar. María del Mar Grandío Pérez, en su libro Adictos a las series define a los fans actuales como "la joya de

\footnotetext{
${ }^{6}$ Fans, principalmente, de cantantes o grupos musicales, que viajan siguiendo sus conciertos, con el deseo de conocerlos personalmente o incluso tener una relación con ellos.

${ }^{7}$ Traducción propia: Durante los últimos 10 años, al menos, las mujeres han realizado más del $90 \%$ de los escritos y los trabajos gráficos, y casi toda la edición de las publicaciones de fans de Star Trek.

${ }^{8}$ Tipo de trabajo realizado por fans de temática homosexual. El término ha quedado reservado para las relaciones entre hombres.
} 
la corona televisiva. Incomprendidos hace unas décadas, relegados a sus aspectos más anecdóticos como su vestimenta o fervor desorbitado y dejados casi en la clandestinidad, los fans son ahora estimados y muy valorados por la industria" (Grandío, 2016: 17). Sin embargo, el fanfiction -y, sobre todo, el femenino- aún conserva connotaciones negativas, o así se percibe incluso dentro de las propias comunidades, como se explica en textos como El desprecio al fanfic: ¿una forma de misoginia?, de Anna Roldós, o en las entrevistas a creadores del fandom que se han realizado en el transcurso de este estudio. Se pone en duda su calidad y su temática, seguramente por centrarse prioritariamente en el mundo de los sentimientos, los romances y las relaciones interpersonales.

Se ha querido destacar a las creadoras de fanfiction hispanohablantes y sus trabajos por la importancia histórica que, como actividad dentro del fandom, tiene la escritura de este tipo de relatos. Siendo la actividad que mayoritariamente desarrollan las mujeres dentro de las comunidades de fans -pues apenas hay hombres escritores-, estos relatos no solo suelen ser el centro neurálgico del fandom encabezado por mujeres, sino que, desde hace años, es la manera en la que ciertos colectivos, en la mayoría de los casos femeninos, se sienten representados en los medios de comunicación. Por otro lado, aunque el tema de las mujeres como escritoras fans ha sido tratado con bastante profundidad en algunos estudios en el mundo anglosajón, no ha sido así en el mundo hispanohablante -posiblemente, por la identificación de las escritoras fans como un todo, sin tener en cuenta su lengua materna-, por lo que este estudio se puede considerar pionero en lo que a ese aspecto refiere.

\section{Objeto y objetivos de la investigación}

Esta investigación tiene por objeto de estudio a las mujeres escritoras de fanfiction hispanohablantes y se aborda desde dos objetivos concretos:

1) Describir y caracterizar a las escritoras hispanohablantes de fanfiction. ¿Quiénes son?, ¿dónde están?, ¿qué hacen?, ¿qué motivaciones las mueven?

2) Explicar la feminización del fanfiction y, de igual manera, de los fandoms a través de las temáticas predominantes.

Se anticipa que el fandom y el fanfiction son una zona segura para que las mujeres puedan desarrollar sus temas de interés, banalizados en otros contextos de la vida.

\section{Metodología}

Se ha utilizado una metodología mixta, cualitativa y cuantitativa, con el fin de encontrar un procedimiento adecuado mediante la triangulación en la recolección de datos de distintas fuentes primarias obtenidas ex profeso para la investigación. Los datos cuantita- 
tivos, se han obtenido a partir de un cuestionario de elaboración propia realizado a fans de los medios de comunicación. Los cualitativos se obtuvieron a partir de trece entrevistas personales, semiestructuradas, a personas que crean contenidos de distintos fandoms.

El cuestionario -que se fraguó como parte de un trabajo académico más amplio- se creó a través de los Formularios de Google, para que fuera más adecuada su difusión por Internet -donde en la actualidad se suele encontrar el mayor número de fans y donde principalmente se distribuyen los fanfictions-. Contaba con 35 preguntas, que buscaban recabar la mayor información posible acerca de los fans, de los miembros de sus diversas comunidades y de los creadores de contenidos que en ellas se encontraban, desde un punto de vista descriptivo y exploratorio.

La muestra fue intencional, centrándose en la posibilidad de llegar al mayor número posible de fans hispanohablantes, por lo tanto, no representativa estadísticamente, ya que el cuestionario se difundió por Internet y fue contestado por aquellos fans que así lo decidieron. No obstante, se considera que el número de fans, -y, en este caso, el número de escritoras de fanfiction- que respondieron la encuesta es suficientemente relevante para dar valor a las conclusiones.

El tuit con el cuestionario fue colgado por primera vez en la red social Twitter el 5 de febrero de $2016^{9}$, con una petición expresa de que se difundiera. El cuestionario llegó a Facebook y, posiblemente, a otras redes sociales, y en Twitter fue retuiteado por cuentas de cadenas de televisión de pago especializadas en ficción y otras dedicadas a diversos productos culturales y sus fans. Cuando se cerró la encuesta definitivamente, un mes después de su publicación, se habían recibido 2406 cuestionarios válidos de personas que se consideraban a sí mismas fans. Del total de estos cuestionarios, 1586 fueron respondidos por mujeres, de las cuales un 40,7 \% -646- eran escritoras de fanfiction.

En el caso de las entrevistas se buscaba, por un lado, conseguir una representación lo más realista posible de los creadores de contenidos hispanohablantes y, por otro, poder conocer y recabar la opinión y la experiencia personal de estos. Así, estos fans creadores pertenecen a diferentes fandoms, disciplinas -escritores de fanfiction, betas ${ }^{10}$, fans artistas, creadores de vídeos, subtituladores...-, nacionalidades -española, mexicana y argentina-, géneros y edades -desde nacidos en 1970 hasta 1998-. Todos ellos son hispanohablantes y 9 tienen una relación muy cercana con el fanfiction. La muestra cuenta con 13 sujetos y se obtuvo buscando creadores y creadoras relevantes dentro de las comunidades de fans. Esto fue por diversos motivos, pero, sobre todo, por su reconocimiento y notoriedad en diferentes comunidades de fans, premios recibidos, número de subscriptores o una larga y prolífera trayectoria dentro de sus respetivos fandoms.

Las entrevistas se hicieron a través de diferentes aplicaciones y redes sociales que permiten realizar videollamadas, como Skype, Facebook, Hangouts e incluso telefónicamente.

\footnotetext{
${ }^{9}$ Los datos recogidos no se pudieron dar a conocer con anterioridad por diversos motivos académicos, pero se considera que aportan una información importante, suficientemente significativa y que no ha perdido validez con respecto a este fenómeno.

${ }^{10}$ También conocidas como betas reader, son una especie de editores que leen una obra escrita, en este caso un fanfiction, de una manera crítica con el objetivo de mejorar la gramática, ortografía y estilo de un texto antes de exponerlo al público.
} 


\section{Resultados}

\subsection{Las mujeres escritoras de fanfiction}

En la "Introducción" se ha desarrollado, sin entrar en profundidad, la idea de cómo la fan mujer, al igual que la escritora de fics mujer, pasó de ser prácticamente invisible a ser la abanderada de algunas comunidades de fans al final del siglo pasado. Si nos acercamos más a la actualidad y, sobre todo, al contexto hispanohablante, todo lo que se ha visto y analizado hasta el momento nos hace vislumbrar que el fandom y el fanfiction lo son en femenino.

De hecho, fue complicado encontrar hombres escritores de fics, o creadores de otro tipo de contenido, dispuestos a participar en las entrevistas realizadas. La complicación radicó, por una parte, en que los alias utilizados normalmente no permiten identificar el género; por otra, en la dificultad para conseguir un contacto fructífero que resultase en la obtención de las respuestas buscadas; pero, sobre todo, en su ausencia dentro de muchas comunidades de fans, algo patente para los propios escritores.

Marion, una de las escritoras de fics entrevistadas más veteranas, comentó: "Yo me he topado con un escritor de fanfic en mi vida, y fue al principio de todo". Haciendo una estimación de su experiencia, se atrevía a comentar que el 99,9 \% de los escritores eran mujeres. De hecho, uno de los pocos entrevistados masculinos y escritor de fanfiction, KalEl el Vigilante, nos hizo partícipes de estas mismas observaciones. "Me sorprendió mucho, hace dos años ${ }^{11}$, cuando empecé a ser consciente de que la producción de fanfic es principalmente femenina, porque nunca había sido consciente de ello". También comentó que, en el año 1994, cuando él escribió su primer relato fan, "era algo completamente heterogéneo, había hombres, mujeres, no había ningún tipo de especialización".

$\mathrm{Al}$ analizar los datos conseguidos con la realización del cuestionario fan, nos encontramos con que estos no se alejan demasiado de esta idea. Según se puede observar (Tabla 1), de las 1586 mujeres hispanohablantes que contestaron el cuestionario, un 40,7\% de ellas -646- eran escritoras, mientras que de los 815 hombres que contestaron, solo un $12,6 \%$ de ellos -103- escribían este tipo de relatos.

Tabla 1. Porcentaje de fans hispanohablantes que escriben fanfiction dependiendo de su género. [Fuente: elaboración propia].

\begin{tabular}{|l|c|c|}
\hline ¿Escribes o has escrito fanfiction? & Mujeres & Hombres \\
\hline Sí & $40,7 \%$ & $12,6 \%$ \\
\hline No & $59,1 \%$ & $87,2 \%$ \\
\hline NC & $0,1 \%$ & $0,1 \%$ \\
\hline N & $100,0 \%$ & $100,0 \%$ \\
\hline
\end{tabular}

${ }^{11}$ Aproximadamente en el año 2017. 
Por otro lado, de ese 40,7 \% de escritoras, un 18,3\% decía no haber publicado nunca sus historias, mientras que, dentro de los escritores un 35\% decidió no publicar. Aunque el cuestionario, evidentemente, no abarca a todos los escritores de fanfic, parece fácil comprender por qué la visión general de todo aquel que conoce el mundo de los fans es que el fanfiction, en la actualidad, es un tema de mujeres.

Para conseguir una radiografía lo mejor delimitada posible sobre esta mayoría de escritoras fans hispanohablantes, se cuenta con las observaciones que se han venido realizando en algunas comunidades virtuales de fans a lo largo de los últimos 20 años, con las respuestas de las 646 escritoras encuestadas y con los comentarios y observaciones de los fans entrevistados.

En primer lugar, se acotará el rango de edad. Las escritoras de fanfiction, principalmente y según el cuestionario realizado, son jóvenes que no han cumplido la treintena. Aunque se encuentran representadas todas las franjas de edad hasta los 54 años, el rango de edad más característico de estas escritoras está entre los 19 y 28 años, con más de la mitad de las encuestadas. La ausencia de escritoras mayores de 55 años puede encontrar explicación en que la incorporación masiva de las mujeres en el fandom en el mundo hispanohablante empezó a partir de finales del siglo pasado, con la democratización de Internet, lo que deja a las mujeres de mediana edad un poco fuera de esta tendencia. Sea como fuere, lo cierto es que las cifras más significativas de escritoras se encuentran entre el rango de edad de los 14 a los 43 años -(Tabla 2)-.

Tabla 2. Edad de las escritoras de fanfiction hispanohablantes. [Fuente: elaboración propia].

\begin{tabular}{|l|c|}
\hline Edad & Escritoras \\
\hline 13 años o menos & $0,8 \%$ \\
\hline De 14 años a 18 años & $16,3 \%$ \\
\hline De 19 años a 23 años & $32,5 \%$ \\
\hline De 24 años a 28 años & $24,5 \%$ \\
\hline De 29 años a 33 años & $13,9 \%$ \\
\hline De 34 años a 43 años & $10,5 \%$ \\
\hline De 44 años a 53 años & $1,4 \%$ \\
\hline Más de 54 años & $0,0 \%$ \\
\hline NC & $0,2 \%$ \\
\hline N & $100,0 \%$ \\
\hline
\end{tabular}

$\mathrm{Al}$ analizar la nacionalidad de estas escritoras -(Tabla 3)-, se ha visto un sesgo muy marcado hacia la nacionalidad española. Este sesgo también estaba presente en la parte de la encuesta dirigida a todos los fans hispanohablantes y no hay posibilidad de saber en cuánto afectó a estos resultados que el cuestionario, aunque fuera difundido a través de la red, se originara en España. 
Tabla 3. Nacionalidad de las escritoras de fanfiction hispanohablantes. [Fuente: elaboración propia].

\begin{tabular}{|l|c|}
\hline Nacionalidad & Escritoras \\
\hline España & $69,0 \%$ \\
\hline México & $12,1 \%$ \\
\hline Argentina & $5,9 \%$ \\
\hline Chile & $3,4 \%$ \\
\hline Venezuela & $1,5 \%$ \\
\hline Colombia & $1,5 \%$ \\
\hline Uruguay & $1,2 \%$ \\
\hline Perú & $1,1 \%$ \\
\hline Costa Rica & $0,9 \%$ \\
\hline Resto de países hispanohablantes & $0,3 \%$ \\
\hline Otros países no hispanohablantes & $0,5 \%$ \\
\hline NC & $0,2 \%$ \\
\hline N & $100,0 \%$ \\
\hline
\end{tabular}

Tabla 4. Últimos estudios realizados por las escritoras de fanfiction hispanohablantes. [Fuente: elaboración propia].

\begin{tabular}{|l|c|}
\hline Estudios finalizados & Escritoras \\
\hline Educación primaria & $2,2 \%$ \\
\hline Educación secundaria & $15,5 \%$ \\
\hline Educación preuniversitaria & $27,7 \%$ \\
\hline Formación profesional & $11,3 \%$ \\
\hline Educación universitaria & $30,0 \%$ \\
\hline Postgrados, doctorados, masters & $13,0 \%$ \\
\hline NC & $0,3 \%$ \\
\hline N & $100,0 \%$ \\
\hline
\end{tabular}

No obstante, aunque el número de escritoras hispanoamericanas no alcanza el número de españolas, ni en la encuesta, ni en lo observado a lo largo de los años, sí que había y hay un gran número de escritoras no españolas muy prolíferas y reconocidas en los diferentes fandoms. De hecho, en la búsqueda de escritoras mexicanas y argentinas para las entrevistas, apenas hubo problemas aparte de los derivados del cambio horario.

La mayoría de las escritoras de fanfic, como se puede observar (Tabla 4), tienen estudios universitarios o los están cursando. Por otro lado, alrededor de un $20 \%$ de ellas ha estudiado o desea estudiar alguna carrera relacionada con las letras -Filología, Literatura, Ciencias de la Información ${ }^{12}$...- para poder dedicarse a alguna profesión que les permita escribir, como la de escritora, guionista, periodista, etc. Casi la mitad, un 44, 4 \%, ya escribía o escribe ficción original además de fanfiction. Y a casi un $37 \%$ de ellas, construir estas historias las ha motivado para seguir escribiendo -(Tabla 5)-.

\footnotetext{
${ }^{12}$ En España las carreras que pertenecían a la especialidad de Ciencias de la Información eran Periodismo, Comunicación Audiovisual y Publicidad y Relaciones Públicas.
} 
Tabla 5. Opciones que más representan a las escritoras de fanfiction hispanohablantes con referencia a sus escritos. [Fuente: elaboración propia].

\begin{tabular}{|l|c|}
\hline Opciones que más te representan en referencia al mundo de los fanfics & Escritoras \\
\hline Ya escribía fanfic antes de saber lo que era. & $40,4 \%$ \\
\hline Me gustaría ser escritora, guionista, periodista... algo que me permita escribir. & $21,1 \%$ \\
\hline Escribo fanfic porque me gusta escribir y así es más fácil llegar a lectores potenciales. & $28,6 \%$ \\
\hline He estudiado o estudiaré Filología, Literatura, Ciencias de la Información... & $19,2 \%$ \\
\hline Escribo o escribía ficción original. & $44,4 \%$ \\
\hline He escrito fanfiction en un idioma diferente al castellano. & $24,0 \%$ \\
\hline Escribir fanfic me motiva a seguir escribiendo. & $36,8 \%$ \\
\hline
\end{tabular}

Aunque ya se tratará el tema de la motivación más adelante, a la hora de querer conocer a estas escritoras, es importante entender que en muchos casos la motivación empieza y acaba dentro de ellas mismas. Como hobby, el escribir fanfiction puede relajar, divertir y entretener, mientras que, como exposición artística es una forma legítima de contar historias, con dos beneficios incluidos. Primero, en un fandom se escribe sobre personajes que ya se conocen y aprecian y, en segundo lugar, da la posibilidad de poder compartir esas historias con personas que las valoran -se cuenta desde el principio con el público objetivo-.

Un buen número de las encuestadas y la mayoría de las entrevistadas tienen aspiraciones literarias. Y dado que el fanfiction es una forma de practicar, y como la mayor parte de ellas, un 40 \%, aún está estudiando -según la encuesta realizada-, es una realidad posible.

Entrando más pormenorizadamente en quiénes son estas escritoras en relación con las comunidades de fans donde escriben, se ha podido observar que más del $60 \%$ lleva formando parte de ellas entre 6 y 15 años.

Como se puede observar (Tabla 6), la diferencia entre las escritoras que llevan siendo fans de 6 a 10 años (algo más de un $37 \%$ ) y las que llevan menos de 5 años (no llega a

Tabla 6. Años que llevan en el fandom las escritoras de fanfiction hispanohablantes. [Fuente: elaboración propia].

\begin{tabular}{|l|c|}
\hline ¿Cuántos años llevas formando parte del mundo de los fans? & Escritoras \\
\hline De 1 a 5 años & $11,8 \%$ \\
\hline De 6 a 10 años & $37,2 \%$ \\
\hline De 11 a 15 años & $25,1 \%$ \\
\hline De 16 a 20 años & $17,2 \%$ \\
\hline De 21 a 25 años & $2,6 \%$ \\
\hline De 26 a 35 años & $1,5 \%$ \\
\hline Más de 35 & $0,0 \%$ \\
\hline Otras respuestas & $3,6 \%$ \\
\hline NC & $1,1 \%$ \\
\hline N & $100,0 \%$ \\
\hline
\end{tabular}


Tabla 7. Productos culturales a través de los cuales las escritoras de fanfiction entran en las comunidades de fans. [Fuente: elaboración propia].

\begin{tabular}{|l|c|}
\hline Producto cultural a través del cual te haces fan & Escritoras \\
\hline Series de televisión & $48,1 \%$ \\
\hline Libros & $27,2 \%$ \\
\hline Películas & $6,5 \%$ \\
\hline Cómics & $3,9 \%$ \\
\hline Videojuegos & $3,4 \%$ \\
\hline Otros & $3,9 \%$ \\
\hline Varios & $4,0 \%$ \\
\hline Todos & $2,8 \%$ \\
\hline NC & $0,2 \%$ \\
\hline $\mathrm{N}$ & $100,0 \%$ \\
\hline
\end{tabular}

un $12 \%$ ) es bastante significativa. Últimamente, se puede escuchar y es un debate abierto en algunas comunidades de fans, que el fandom como se conocía a nivel hispanohablante ya ha vivido su mayor momento de esplendor. ¿Es posible que la escritura de fanfic sea una actividad en decadencia tras años teniendo una gran importancia en cualquier fandom hispanohablante?

Con respecto a los productos culturales que más atraen a las escritoras, según se observa (Tabla 7), la mayoría de las escritoras entran en el mundo del fandom a través de las series de televisión; este es el caso de casi la mitad de las encuestadas. Claramente, la idea de una emisión constante con paradas más o menos largas ayuda a la creación, dándole un espacio en el tiempo. En un segundo puesto, con más de un cuarto de las encuestadas, se encuentran los libros. Teniendo en cuenta que la gran afición de nuestras encuestadas es escribir, este dato apenas necesita explicación.

Con respecto a los productos culturales más seguidos por los escritores, el género parece un tema relevante, así que se ha realizado una tabla cruzada -(Tabla 8)- para poder analizar más pormenorizadamente los datos recibidos respecto a las preferencias de las escritoras de fanfic.

Los únicos productos culturales que parecen despertar un interés parecido tanto en hombres como en mujeres escritores son los originarios de Marvel $^{13}$ y la serie Doctor Who (BBC, 1963-1989, 2005-actualidad). Ya que los porcentajes obtenidos en los productos de los que los encuestados escritores hombres y mujeres son fans hacen dos listados bastante diferenciados.

Lo más llamativo es claramente el gran fenómeno fan que ha desencadenado el universo de Harry Potter en las escritoras de fanfic, que casi llega a un $70 \%$ de las encuestadas. Con el resto de los productos culturales más seguidos, los escritores se mueven en porcentajes que no superan el $35 \%$ de los encuestados, ni en hombres, ni en mujeres.

\footnotetext{
${ }^{13}$ Marvel Entertainment es una empresa estadounidense de entretenimiento conocida por desarrollar productos cinematográficos, televisivos y editoriales alrededor de un gran número de superhéroes de cómics.
} 
Tabla 8. Comparativa de productos, personajes o universos de los que son más fans los escritores de fanfiction según género. [Fuente: elaboración propia].

\begin{tabular}{|l|c|c|}
\hline ¿De qué eres o has sido fan? & Escritoras de fics & Escritores de fics \\
\hline Universo Harry Potter & $68,3 \%$ & $30,1 \%$ \\
\hline Universo Marvel & $31,3 \%$ & $34,0 \%$ \\
\hline Universo Star Wars & $13,3 \%$ & $28,2 \%$ \\
\hline Universo Juego de Tronos & $12,5 \%$ & $19,4 \%$ \\
\hline Universo El Señor de los Anillos & $12,8 \%$ & $22,3 \%$ \\
\hline Expediente X & $10,7 \%$ & $1,9 \%$ \\
\hline Doctor Who & $15,0 \%$ & $14,6 \%$ \\
\hline Universo DC & $7,4 \%$ & $11,7 \%$ \\
\hline Universo Los juegos del hambre & $9,0 \%$ & $3,9 \%$ \\
\hline Universo Sherlock Holmes & $11,0 \%$ & $1,9 \%$ \\
\hline
\end{tabular}

Al contrario que en el caso de Harry Potter, se puede ver que Marvel tiene un porcentaje muy similar de público, tanto femenino como masculino, en el mundo de los escritores, pero no es lo que sucede normalmente. Tanto es así que en el "top 10" femenino entran productos que no aparecen en el masculino. Por ejemplo, el personaje de Sherlock Holmes, que está en el puesto número siete, y las series de ciencia ficción Expediente X (The X-Files, Fox, 1993-2002, 2016-2018), en el número ocho, y Supernatural (Warner Bros. Television, 2005-2020), en el número nueve, con un 8,5 \% de las escritoras como fans de este producto.

Si se observa este listado de productos más admirados por las escritoras de fanfiction y se compara con el número de escritos encontrados en, por ejemplo, el sitio Fanfiction.net ${ }^{14}$-uno de los que más relatos tiene archivados en la web-, se puede ver un cierto paralelismo.

Harry Potter no solo es el producto con más escritos de la sección de libros, con 825000 fanfictions, al momento de la investigación, sino también de toda la web. En la sección de libros también se pueden encontrar las trilogías de El Señor de los Anillos, en cuarta posición, con 57000 historias, y de Los juegos del hambre, en quinta posición, con 45 900. En el puesto 14, están los libros de Canción de hielo y fuego, con 9400 relatos en su haber -y otros 9400 en el apartado de series de televisión con Juego de Tronos (Game of Thrones, HBO, 2011-2019)-.

Las películas del universo Star Wars y las de Los Vengadores -uno de los productos más visibles de Marvel- son las que tienen mayor cantidad de fanfiction en la web. En el caso de Star Wars, 55 400; en el de Los Vengadores, 49 400. En la categoría de series de televisión, la primera de la lista es, con 126000 relatos, Supernatural; en tercer lugar se encuentra Doctor Who, con 76100, y en cuarto lugar Sherlock (BBC, 2010-2017), con 60200 -este personaje también cuenta con 4100 fics en el apartado de libros y 2000 en el de películas-.

\footnotetext{
${ }^{14}$ https://www.fanfiction.net/
} 
Teniendo en cuenta que ya en 2002 el $80 \%$ de los usuarios de esta web eran mujeres (Roldós, 2019), los gustos de las escritoras, tanto a la hora de disfrutar como a la hora de escribir, parecen claros.

\subsection{El fanfiction feminizado}

Con todo lo expuesto hasta el momento, como ya se ha anticipado en este estudio, no cabe duda de que en la actualidad las mujeres son mayoría en los espacios donde el fanfiction es una actividad importante. Lo que no es tan patente es si las mujeres fans ganaron ese espacio al ser mayoría o si los hombres fans se fueron de él porque las temáticas desarrolladas no les gustaban y buscaron otros puntos de encuentro.

El fanfiction actual habla principalmente de cuestiones que parecen interesar más a las mujeres: relaciones románticas, interpersonales... En estos relatos se desarrollan personajes, se solventan conflictos y, sobre todo, se cierran historias que quedaron o están abiertas en la obra madre. Las mujeres cuentan historias que las representan y les interesan en más de un sentido, y buscan una visibilidad en los medios que no siempre han tenido.

\subsubsection{El ship ${ }^{15}$ y las shippers ${ }^{16}$}

Al igual que cada serie, libro, película... tiene un público objetivo, así el fandom y sus respectivas actividades también lo tienen. De hecho, Jenkins afirma que, en general, hombres y mujeres buscan cosas diferentes en los productos culturales y, de igual manera, aportan cosas diferentes.

Con respecto a la serie Twin Peaks (ABC, 1990-1992), Jenkins hacía la siguiente reflexión:

Es posible que los fans masculinos se sintieran atraídos por la serie porque permitía hacer especulaciones sintagmáticas. De hecho, estos fans deseaban más complejidad narrativa que la que Lynch podía ofrecer, pues proponían elaboradas teorías conspiradoras o construían "casos irrefutables" contra sospechosos tan improbables como Dale Cooper, Andy, el ayudante del sheriff, o el doctor Hayward. Las fans mujeres abrazaron sus dimensiones paradigmáticas, en especial, la amistad entre el sheriff Truman y el agente Cooper, que es el eje de las obras de ficción sobre Twin Peaks escritas por fans. Cuando los fans hombres escribían sus propios "guiones", se centraban más en la creación de nuevos enigmas narrativos que en desarrollar de forma más detallada las relaciones entre los personajes. (Jenkins, 2010:136)

\footnotetext{
${ }^{15}$ Término que se utiliza para nombrar una pareja con una posible relación romántica que interesa a los fans.

${ }^{16}$ Término que define a todos aquellos fans que tienen un ship. En un principio definía a los fans de Expediente Xque defendían una relación romántica entre Mulder y Scully. En la actualidad es un término aplicable a los fans de cualquier ship. 
Respecto a otra serie con un gran fenómeno fan y, sobre todo, con una gran cantidad de fanfiction en su haber, Expediente X, la creadora del libro Expediente X. En honor a la verdad comentaba:

Hay diversos trabajos académicos que centran su atención, sobre todo, en la notable presencia de mujeres en las diversas comunidades $x$-philes de la red, debido tanto al atractivo feminista de Scully como a su complicada relación con Mulder. Según parece, mientras los hombres han debatido mayoritariamente cuestiones centradas en la ciencia y la tecnología de la serie, las mujeres se han interesado más por las relaciones personales dentro de ella. (Martín, 2006: 41)

Y lo cierto es que, al hacer un análisis superficial en las páginas donde a finales de siglo pasado se podían encontrar relatos de la serie Expediente X, más de un $95 \%$ de ellos habían sido escritos por mujeres y eran relatos shippers, con trasfondo paranormal o sin él. Por lo general, los fans hombres, la mayoría noromos -fans que no querían que la relación de Mulder y Scully fuera más allá de la amistad-, escribían relatos más centrados en la temática paranormal. Parece ser que, en general, eran cosas diferentes las que enganchaban a la serie a hombres y mujeres, y las que los llevaban al fandom, pero fueron las mujeres las que acabaron ocupando el mayor espacio en las comunidades online.

Por lo general, como ya se ha establecido, los hombres fans no suelen sentir interés por los productos que se basan en las relaciones amorosas, así que no forman parte de los fandoms monopolizados por esta temática. Al mismo tiempo, las mujeres a las que el fandom les interesa principalmente para shippear no se involucran tanto si no ven una posibilidad clara de retroalimentación a su trabajo o a sus intereses.

Por ejemplo, un caso muy reciente es el de los relatos que se pueden encontrar sobre la serie El Ministerio del Tiempo (TVE, 2015-2020), cuyos escritores son hombres en un porcentaje bastante alto. Estos fanfics se centran principalmente en la ciencia ficción y en la historia de España. Apenas hay trabajos relacionados con el ship Julián y Amelia -Jumelia-, que en la primera temporada se podía considerar como el oficial. Aunque, por otra parte, sí que se pueden encontrar historias donde la amistad de Julián y Alonso -dos personajes heterosexuales en la serie- va más allá de la del simple compañerismo.

\subsubsection{El slash y el femslash ${ }^{17}$}

La temática slash, tanto en los fanfictions como en el resto de las actividades del fandom, comenzó a desarrollarse en los años 70, por mujeres, en algunas comunidades angloparlantes de fans de Star Trek. Estas mujeres, fans y creadoras de contenido, empezaron a percibir que la relación entre los personajes del capitán Kirk y el señor Spock iba más allá de la amistad. En ningún momento, en la serie, se hablaba de la posible bisexualidad u homosexualidad de estos personajes, así que estas historias vulneraban las caracterís-

${ }^{17}$ Tipo de trabajo realizado por fans de temática lésbica. 
ticas originales y se consideraban "malas". Sea como fuere, los relatos de temática slash se quedaron en el fandom de Star Trek, con un buen número tanto de escritoras como de lectoras, y la temática slash ha llegado hasta nuestros días, cada vez más reforzada y evolucionada, aunque no sin cierta polémica.

El slash, (...) es simplemente una forma más de escritura fan entre muchas otras y, por tanto, uno puede formar parte de un grupo de fans sin desarrollar una afición por el slash y sin leer ninguna de sus historias. Sin embargo, el slash constituye un género importante en las publicaciones creadas por fans, y posiblemente sea la contribución más original de estos grupos al ámbito de la literatura popular. (Jenkins, 2010:218)

En cualquier página de fanfiction es posible encontrar numerosos relatos de temática slash, con dos personajes masculinos heterosexuales como protagonistas y con prácticamente ninguna posibilidad de convertirse en canon $^{18}$ en el producto original. Por poner algunos ejemplos, en el fandom del universo Harry Potter se puede encontrar a Draco Malfoy con Harry Potter -Drarry- o a Sirius Black con Remus Lupin -Wolfstar-; en el universo de Marvel, a Steve Rogers con Bucky Barnes -Stucky- o a Thor con Loki -Thorki-; en la serie Supernatural, a Dean con Sam -Wincest- o Dean con Castiel -Destiel-; o en el universo que rodea al personaje de Sherlock Holmes, a Sherlock con Watson. Las principales consumidoras y creadoras de estas obras son mujeres. Como pasa con otro tipo de temática, el slash no se desarrolla en todos los productos culturales y hay algunos que carecen completamente de él. De hecho, hay escritoras y lectoras que han estado muchos años en uno u otro fandom y nunca han escrito o leído slash o femslash.

De todos modos, la siguiente pregunta puede parecer obvia: ¿qué hace a tantas mujeres escribir historias homosexuales con personajes masculinos?

Antes de entrar en esta cuestión se hace necesario realizar un pequeño paréntesis para comentar el femslash. Después de todo, la evolución del slash hacia este, en relación con la comunidad LGTB+ femenina cobra sentido por la necesidad de esta comunidad de verse representada en la cultura popular que solo recientemente comenzó a darles cierta cabida. Así se expresaba a este respecto una de las entrevistadas, Sixphanel: "Lo que leo y lo que escribo es todo femslash y supongo que es una vía de buscar lo que no veo normalmente en lo que consumo, como no aparece de forma común en la televisión o en el cine. Es verme más representada a mí en ciertas cosas".

De hecho, en la actualidad y desde hace unos años, el material femslash está viviendo una época de bonanza tanto canon como no canon. Se puede decir que esta dinámica empezó con la serie Xena: la princesa guerrera (Xena: Warrior Princess, Renaissance Pictures, 1995-2001), encarnada en los personajes de Xena y Gabrielle, cuya posible relación romántica nunca llegó a ser canon, aunque diera para mucha literatura tanto dentro como fuera del fandom.

\footnotetext{
${ }^{18}$ Algo que es cierto en el universo de una serie, película, libro, cómic... porque así lo han decidido sus creadores.
} 
Al hablar de series hispanohablantes más recientes, se puede decir que desde hace ya bastantes años, algunas producciones incluyen a parejas románticas de mujeres que suelen ser muy aplaudidas y seguidas por sus fandoms y que han dado mucho fanfiction a sus respectivas comunidades. Algunas de las más recientes pueden ser Maite y Camino -Maitino- en Acacias 38 (TVE, 2015-actualidad), Luisa y Amelia -Luimelia- en Amar es para siempre (Atresmedia, 2013-actualidad) o Julieta y Valentina -Juliantina- en Amar a muerte (W Studios, 2018-2019). Estos personajes tienen la mayor parte de la atención del fandom de estas series y el mayor número de actividades fans, lo que deja patente lo importante que es la representación. No se está insinuando que todos aquellos que disfrutan de esos ship LGTB+ lo sean, pero, al crear fandoms alrededor de esta temática, esos lugares se convierten en sitios seguros para que la comunidad LGTB+ pueda vivir su identidad libremente y sentirse representada, algo que llevan años intentando conseguir.

De igual manera, la mujer busca una representación fidedigna en los medios de comunicación que aún hoy no siempre encuentra. Como ya se ha expuesto, uno de los valores positivos con los que contaba la serie Expediente $X$, y con el que obtuvo mucho público femenino, fue la presencia del personaje de Dana Scully, una mujer joven, íntegra y profesional. Su personaje fue el referente para muchas jóvenes en los 90. De hecho, recientemente se ha validado el conocido como "Efecto Scully", que defendía que muchas jóvenes que se criaron con Dana Scully como referente se decidieron, gracias a ello, a estudiar carreras científicas.

Las mujeres llevan años buscando su espacio en los medios y, al no sentirse completamente representadas, el fandom y el poder contar sus propias historias les dieron y dan la oportunidad de encontrar su lugar. Y, curiosamente, esta misma premisa puede explicar, en parte, el amplio desarrollo del slash masculino escrito por mujeres.

En su artículo Male in pregnet: ¿Por qué las mujeres escriben sobre embarazo masculino?, Lucía y Lourdes Castillo Pacheco (2013:119) hablan de ciertos puntos que deben tenerse en cuenta.

Por un lado, está la posibilidad de apropiarse de la historia, como necesidad de participar en el imaginario social donde antes no se había podido, algo que el fandom, el fanfic y el anonimato permiten sin problema -no solo en la temática slash-. Por otro lado, las mujeres escriben este tipo de relatos porque se puede hacer. Hay libertad para experimentar con conceptos alternativos sin que la escritora sea expuesta o juzgada personalmente. Aunque es cierto que hay temas que siguen siendo muy controvertidos e incluso mal vistos dentro del fandom, estas suelen ser comunidades abiertas; o, en todo caso, las diferentes temáticas se pueden mantener separadas.

Así mismo, y volviendo al tema de la identificación y representación, aunque en los últimos años cada vez hay más personajes femeninos que intentan parecer reales en las ficciones mediáticas, no siempre se consigue de una manera satisfactoria y, desde luego, no era así cuando nació y se desarrolló el fanfic slash masculino.

El papel de la mujer no ha crecido a la par de la mujer real o no refleja las aspiraciones que tienen las mujeres fans. Por eso, las mujeres no se identifican con las mujeres de la pantalla; se identifican con el héroe masculino, que es quien tiene poder en la ficción y, si ese 
héroe es tan parecido a ellas (nosotras), entonces es posible que se enamore de sus compañeros o de los otros hombres del elenco que son interesantes como personajes; y no de la protagonista femenina, que es sosa y provoca poca empatía. (Castillo y Pacheco, 2013:119)

Y para finalizar con este punto, está más que comprobado que lo que más le gusta a la escritora de fanfic -más que contar una historia en sí- es, en general, el desarrollo de los personajes, sus pensamientos, sus sentimientos, sus sensaciones. Así que no parece raro pensar que las escritoras de slash exploren la sexualidad masculina en busca de esos sentimientos y necesidades, en busca del hombre que no tema expresarlos. Si durante años todos los productos mediáticos han estado masculinizados, ¿por qué no intentar "feminizar" al héroe?

\subsection{3. ¿Por qué la mujer escribe fanfic?}

El fanfic no aporta ninguna clase de retribución económica, la motivación para escribirlo y desarrollarlo tanto, cuando ni siquiera es una actividad bien vista socialmente fuera del fandom, tiene que ser muy fuerte para haber conseguido acaparar espacios antiguamente masculinos. 0 , tal vez, contar con esos espacios seguros es parte de la motivación y de la feminización de las comunidades de fans. Desde luego, la idea de crear comunidad es muy importante para las mujeres fans en general y, en particular, para las escritoras. También parece muy importante, y para tener en cuenta, la temática predominante. Como ya se ha expuesto, a las mujeres parece que les gusta escribir sobre las relaciones humanas y los fandoms reflejan esa preferencia.

Sin embargo, uno de los principales motivos por los que se escribe fanfiction y no otra cosa parece ser la frustración. "Después de todo, el mundo de los fans nace de un equilibrio entre la fascinación y frustración: si no nos fascinaran los contenidos mediáticos, no tendríamos ningún deseo de involucrarnos en ellos; pero, si no nos frustraran en algún grado, no sentiríamos el impulso de reformularlos y rehacerlos" (Jenkins, 2008:245).

El concepto de fanfiction va "ligado preferentemente al de ficciones en el sentido de textos abiertos y especulativos" (Martos Núñez, 2006: 65), que sean susceptibles de ser continuados.

La cultura de los fans refleja tanto la fascinación del público por los programas como la frustración de los fans por la incapacidad de los productores de contar el tipo de historias que quieren ver. Los escritos de los fans reflejan claramente la dualidad de esta respuesta: los fans escritores no reproducen el texto principal, sino que lo reescriben, corrigiendo o rechazando los aspectos que no les satisfacen, desarrollando intereses que no han sido suficientemente explorados. (Jenkins, 2010: 190)

Las respuestas recibidas en las entrevistas realizadas no hacen más que reafirmar esta idea de que las escritoras de fanfic se sienten motivadas a la hora de escribir y buscan 
dar cierre a las historias que más les gustan ante la frustración que conlleva que el propio producto cultural no lo haga. Por un lado, y como comenta Marion, se quieren "rellenar huecos que el fandom no te está dando", normalmente, desde el punto de vista del ship. Sra. Cheg desarrolla esta idea y nos pone en situación: es estar "tan frustrada con el ship que voy a montar mi propia historia. Ya que no me lo dan los guionistas, me lo voy a montar yo". Justa y curiosamente, esta motivación, que tanto aporta en un fandom altamente feminizado, parece que aleja a otro tipo de fans -como ya hemos visto-. KalEl el Vigilante nos comenta que el ship "evitó que entrara muy a fondo -en el mundo del fanfic-. Ya sabía que existía, pero buscaba, quizás, algo más profesional. Los textos ya escritos por gente que evitaba esos excesos juveniles y que intentaba explicar historias".

Respecto al tema de la motivación, cabe destacar otra idea: la del reconocimiento.

Las mujeres que tienen trabajos de poco prestigio o que son amas de casa pueden obtener un reconociendo nacional e incluso internacional como escritoras y artistas fans: la publicación de obras puede convertirse en una fuente de estatus, un estatus no reconocido por los sistemas sociales y económicos dominantes, pero muy gratificante a nivel personal (Jenkins, 2010:186)

Esta idea puede que haya quedado un poco desactualizada porque, como ya se ha visto, en el contexto hispanohablante de hoy, el mayor número de escritoras son menores de 30 años y estudiantes universitarias, pero el fondo se mantiene intacto. El reconocimiento es un arma poderosa y la vida actual tiende a ser frustrante y alienante. Para una persona, tener el claro convencimiento de que a otra persona le gusta lo que hace y lo valora es una fuente de motivación muy importante. Además, no hay escritores sin lectores... y, aunque cada vez hay menos retroalimentación dentro del mundo del fanfiction, los like y las visualizaciones todavía estimulan y alegran a una escritora, o así lo han comentado las entrevistadas.

\section{Conclusiones}

El fanfiction se puede considerar, en parte, como un lugar de encuentro para las minorías que normalmente no se perciben representadas en los medios de comunicación actual y que, gracias al fandom, han creado sus propios canales de comunicación y su manera personal de transmitir historias. A finales del siglo pasado, Henry Jenkins ya dirimía que, por ejemplo, los relatos de temática slash habían conseguido crear canales de comunicación entre mujeres lesbianas, bisexuales y heterosexuales, proporcionando unas condiciones idóneas para el dialogo en torno a la creación social de género (Jenkins, 2010:253).

No hay un solo fandom, hay muchos, y cada pequeña comunidad de fans dentro de otra más grande se ha convertido en un lugar seguro donde las mujeres pueden desarrollar sus historias y compartirlas en busca de representación y prestigio, aunque sea entre iguales. Aun así, no se puede obviar que la escritura de estos relatos es un trabajo, que puede ser muy exigente, pero que no está remunerado más allá de la motivación personal 
o del apoyo del grupo. Que el mayor grueso de estos narradores sean mujeres no se puede entender como una simple casualidad, y puede estar relacionado con el hecho de que históricamente han sido las mujeres las más acostumbradas a realizar trabajos no remunerados, como el de ama de casa, con mayor o menor reafirmación personal.

El fanfiction nunca ha tenido una visibilidad positiva, incluso dentro de algunas comunidades de fans. Esto es particularmente cierto con aquel abanderado por mujeres, que se centra en relaciones amorosas y que ha sido acusado de ser un producto de mala calidad o incluso de ser simplemente porno para mujeres. No obstante, los relatos lemon ${ }^{19}$ o smuth ${ }^{20}$-het ${ }^{21}$, slash, o femslash (los que más literatura académica han proporcionado)solo son un porcentaje más o menos alto dentro de todos los fanfics. De las 825000 historias de Harry Potter que hay en Fanfiction.net, 160000 tienen la calificación M -para mayores de 16-, lo cual no llega a ser un $20 \%$. En español, son 9500, un $16 \%$ del total de las obras en este idioma.

Del fanfiction femenino parece molestar la temática. El problema es el ship, del tipo que sea, el cual ya apartó a los hombres en un grado muy amplio. Sin embargo, no hay duda de que las mujeres prefieren leer y escribir historias centradas en los sentimientos, donde se puedan sentir representadas en el ámbito emocional. Esto se mantiene incluso en historias donde el amor surge entre dos hombres, se muestre sexualmente o no.

Hay fanfictions estupendos, buenos, malos y horrorosos, al igual que en todos los géneros literarios; sin embargo, el juicio por defecto suele ser que el fanfic es literatura de mala calidad, pero es necesario tener muchas variables en cuenta y alejarse de la generalización. En primer lugar, la edad de las escritoras. Un gran número de escritoras hispanohablantes empiezan a escribir fanfiction alrededor de los 14 años. No es posible pedir una gran calidad literaria cuando se empieza a desarrollar una actividad a tan pronta edad. En muchos casos, y si así lo desea la escritora, el fanfiction se convierte en una especie de taller literario donde experimentar y aprender arropada por la comunidad. En esta labor ha sido muy importante la figura del beta con el paso de los años.

Según el cuestionario realizado, las mujeres hispanohablantes siguen escribiendo hasta bien entrados los 40 años y, en general, tienen formación superior. De hecho, entre sus filas, hay un gran número de mujeres que desean o han deseado escribir de una manera profesional, y se han formado o se quieren formar para ello, "practicando" en el mundo de fanfic. Si se toman en cuenta los datos que se han recolectado en la encuesta, casi un $20 \%$ ha recibido o recibirá formación literaria o narrativa. Sería muy atrevido pensar que no hay productos de calidad en una comunidad tan amplia, con una producción tan extensa. Repetiremos el dato: en una sola página -Fanfiction.net-, en un solo fandom -Harry Potter-, 825000 fanfiction, 58000 en castellano. Es importante comentar que las escritoras hispanohablantes no solo escriben en español -de hecho, cada vez se tiende más a la escritura en inglés, provenga de donde provenga el escritor. Casi una cuarta parte de las encuestadas dice haber escrito en un idioma diferente al español-.

\footnotetext{
${ }^{19}$ Tipo de fanfic que muestra relaciones sexuales dentro del contexto de una historia desarrollada.

${ }^{20}$ Tipo de fanfic que muestra relaciones sexuales sin contexto previo.

${ }^{21}$ Tipo de trabajo realizado por fans de temática homosexual heterosexual.
} 
Concluyendo, aunque se puede afirmar que el fanfiction femenino trata prioritariamente sobre temas amorosos o sexuales, no es una afirmación totalitaria. Todo tipo de relaciones son importantes: las amistosas, las familiares, el tratamiento de traumas... El desarrollo de los personajes suele ser lo prioritario. De la misma manera, estas comunidades de escritoras que se crean a través de estos relatos forjan lazos de compañerismo y amistad entre sus miembros. Generan espacios donde se puede hablar de todo, o casi todo, lo que los convierte en lugares seguros y de desarrollo. Puede ser que aquello que ha llevado a tanta mujer al fanfiction es lo que ha separado a la otra mitad de la población: el que haya un espacio para todo y para todos.

\section{Bibliografía}

ABAD Ruiz, BÁRBARA. (2011). Fanfiction: fomento de la escritura creativa a través de las formas de literatura emergentes. Revista Electrónica De Estudios Filológicos. TONOS, 21. Recuperado de http://www.um.es/tonosdigital/znum21/secciones/tritonos1-fanfiction.htm

Bacon-Smith, CAMiLle. (1986). Spock among the women. The New York Times. Recuperado de https://www.nytimes.com/1986/11/16/books/spock-among-the-women.html

BACON-SMITH, CAMILLE. (1992). Enterprising women: television fandom and the creation of popular myth. Philadelphia: University of Pennsylvania Press.

Castillo Pacheco, lucia Amapola y Castillo Pacheco, lourdes Eugenia. (2013). Male in pregnet: ¿Por qué las mujeres escriben sobre embarazo masculino? En Lourdes Pacheco Ladrón de Guevara, Laura Isabel Cayeros López, Fabiola González Román, Arturo Murillo Beltrán, María del Refugio Navarro Hernández, Elena Susana Pont Suárez y Dalinda Sandoval Acosta (coordinadores), La equidad de género en el desorden de la violencia. Nuevos y viejos escenarios. Nayarit: Universidad Autónoma de Nayarit y Fundación Universitaria Andaluza Inca Garcilaso para eumed.net, (1aa ed., pp. 117125). Recuperado de http://www.eumed.net/libros-gratis/2013b/1338/index.htm

Cruz Martín, AnNA. (2016). El fenómeno de la fanfiction (trabajo de grado). Universitat de Girona, Girona, España. Recuperado de https://dugi-doc.udg.edu/handle/10256/13312

Díaz Agudelo, Jenny Natalia (2009). Formas emergentes de la literatura: El fanfiction desde los estudios literarios. (Trabajo de Grado). Pontificia Universidad Javeriana, Colombia.

FanFiction.net. (s. f.). Recuperado de https://www.fanfiction.net/

FANFITECA EXPEDIEnTE X. (s. f.). Recuperado de http://lafanficteca.net/

Fernández Mallo, Agustín. (2008). Las fanfictions y el Centro de los Tiempos. El País. Recuperado de http://elpais.com/diario/2008/12/13/babelia/1229128770_850215.html

Francis, Conseula y Piepmeier, Alison. (2011). My Hair Stood on End!: Talking with Joanna Russ about Slash, Community, and Female Sexuality. Journal of Popular Romance Studies, volume 1 (2010-2011), issue 1.2. Recuperado de: http://www.jprstudies. org/2011/03/interview-joanna-russ/ 
García Miranda, Carlos. (2015). Fanfiction: Harry Potter, porno y copyright. El Huffington Post. Recuperado de: http://www.huffingtonpost.es/carlos-garcia-miranda/ fanfiction-harry-potter-p_b_6507740.html

Geena Davis Institute on Gender in Media. (2020). The Scully Effect: I Want to Believe in STEM. Recuperado de https://seejane.org/research-informs-empowers/the-scullyeffect-i-want-to-believe-in-stem/

Grandío PÉrez, María del MAR. (2016). Adictos a las series. 50 años de lecciones de los fans. Barcelona: Editorial UOC.

Guerrero, Mar. (2015). Historias más allá de lo filmado: Fan fiction y narrativa transmedia en series de televisión. (Tesis doctoral) Universitat Pompeu Fabra, Barcelona, España.

Hellekson, Karen y Busse, Kristina (2014). The Fan Fiction Studies Reader. Iowa: University of Iowa Press.

Jenkins, Henry. (2008). Convergence culture. Barcelona: Paidós.

Jenkins, Henry. (2010). Piratas de Textos. Fans, cultura participativa y televisión. Madrid: Paidós Comunicación.

Kozinets, RoBert V. (2001) Utopian Enterprise: Articulating the Meaning of Star Trek's Culture of Consumption. Journal of Consumer Research, January 2001. Recuperado de https://www.researchgate.net/publication/267923074_Utopian_Enterprise_Articulating_the_Meaning_of_Star_Trek's_Culture_of_Consumption

Martín Alegre, SARA. (2006). Expediente X. En honor a la verdad. Madrid: Alberto Santos Editor.

MARTos NúÑEZ, EloY. (2006). “Tunear” los libros: series, fanfiction, blogs y otras prácticas emergentes de lectura. OCNOS, 2, 63-77.

Morán Rodríguez, CARMEn. (2007). Li(nk)teratura de kiosko cibernético: Fanfictions en la red. Cuadernos de Literatura, 12 (23), 27-53.

Rubio-Hernández, María del Mar y López-Rodríguez Francisco Javier. (2012). El fan fiction de temática homoerótica inspirado por productos audiovisuales. Una aproximación desde la narrativa. Revista Comunicación, №10, Vol.1, año 2012, 1183-1198.

Roldós, AnNA. (2019). El desprecio al fanfic: ¿una forma de misoginia? Recuperado de https://lanaveinvisible.com/2019/05/14/el-desprecio-al-fanfic-una-forma-de-misoginia/ 